\title{
Millimeter and Submillimeterwave Receivers
}

\author{
T.G. Phillips \\ California Institute of Technology 320-47 \\ Pasadena, CA 91125, U.S.A.
}

\section{Introduction}

Various types of receivers can be used for millimeter and submillimeterwave astronomy. The choices are amplifiers or mixer detectors. For the millimeter band, in the past, maser and paramp devices have been successfully used, but in the last few years HEMT amplifiers have proved to be the best option up to about $40 \mathrm{GHz}$, because they are inexpensive, quite low noise (about $1-2 \mathrm{~K} / \mathrm{GHz}$ ), stable and wideband. However, currently, above $\sim 40 \mathrm{GHz}$ the best performance is obtained from mixer receivers and this review will address that topic only. When used in either interferometers or in single dish spectroscopy, the receivers measure simultaneously the amplitude and phase of the astronomical signal and are therefore fundamentally limited by the quantum noise inherent in the measurement process, which increases linearly with frequency. A receiver which achieves a noise temperature within a factor of 10 of this limit is considered to be well optimized, so that the usual criterion for the noise temperature characterizing a single side-band receiver is that it should be $\$ 10 h \nu / k$ or equivalently \$ $0.5 \mathrm{~K} / \mathrm{GHz}$.

There are several types of mixer receivers which are used:

a) Schottky Diode receivers: These were the receivers used to develop millimeterwave astronomy, first as room temperature devices (Penzias \& Burrus, 1973 ) and then with improved performance when cooled to $20 \mathrm{~K}$ or so (Weinreb $\&$ Kerr, 1973). The devices use a gold dot to form a Schottky diode barrier on a gallium-arsenide substrate and have been improved for work in both the millimeter and submillimeter bands over many years (e.g. Crowe et al., 1992).

b) Superconducting Tunnel Junction receivers: For much of the frequency range these have taken over from Schottky diodes. There are various possible types; Superconductor-Insulator-Superconductor (SIS) receivers, which use photon assisted quantum mechanical tunneling of quasiparticles from one electrode to the other, through an insulating barrier; Superconductor-Insulator-Normal metal (SIN) and Super-Schottky receivers, which are similar to SIS, but have only one superconducting electrode; and Josephson effect mixer receivers, which use the tunneling of electron pairs. Of these various types, the most successful have been SIS (e.g. Tucker \& Feldman, 1985; Phillips \& Woody, 1982).

c) Fast Bolometer or Photodetector receivers: Since these are square-law detectors they can act as mixers, if the electronic response is fast enough to allow a useful IF range. Earlier a low noise indium-antimonide hot-electron bolometer receiver was used successfully (Phillips \& Jefferts, 1973), with limited 
IF bandwidth ( $\sim \mathrm{MHz})$, but now there is the possibility of a two dimensional electron gas gallium-arsenide version (Yang et al., 1991).

Of the various types of mixer receivers discussed above, the lowest noise in the 40-800 GHz range is from SIS receivers. These are now easy to manufacture and are reliable in use. The upper frequency limit is roughly set by the energy gap $(2 \Delta)$ of the superconductor, which is about $700 \mathrm{GHz}$ for niobium. For frequencies above $800 \mathrm{GHz}$, Schottky diode receivers are currently superior (Crowe et al., 1992; Blundell \& Tong, 1992). However, work on higher $2 \Delta$ SIS junctions, Josephson effect mixers and fast bolometers is continuing for that range. Given the dominance of SIS receivers over most of the range accessible to ground based telescopes, the rest of the discussion will be concentrated on SIS detectors, with also a short discussion of some new results on Josephson devices.

\section{SIS Detectors}

\section{A Physics of SIS Receivers}

The basic physical effect of photon assisted single quasi-particle tunneling was discovered by Dayem and Martin (1962), but first developed for astronomy applications by the Bell Labs/Caltech and Berkeley groups (Dolan, Phillips \& Woody, 1979; Richards et al., 1979), taking advantage of the small area junction fabrication technology developed for superconducting computer circuits. The progress was aided enormously by the excellent theory simultaneously developed by Tucker (1979). Figure 1 describes the underlying physics of the detection process.

(c)

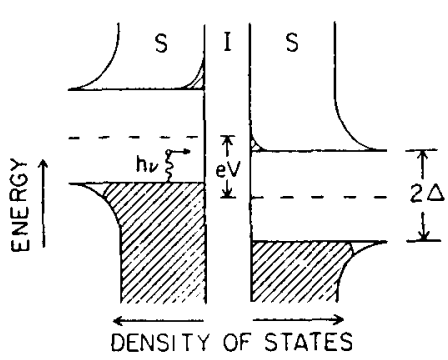

(b)

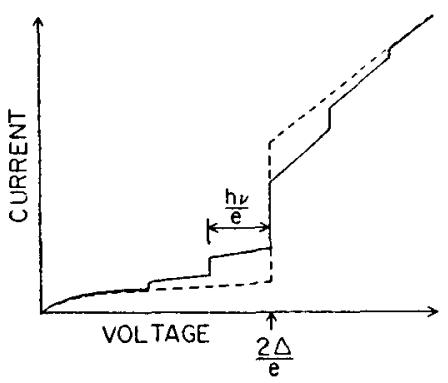

Figure 1: (a) Schematic diagram of density of states. (b) I-V relation, with and without $R F$ power

The left half (a) shows the density of allowed states as a function of energy for electrons above and below the energy gap $(2 \Delta)$ for the two superconducting electrodes, separated by the thin $(\sim 20 \AA)$ insulator. If a variable voltage bias is applied to electrodes, an increase of tunneling current occurs at the value $V=2 \Delta / e$ (the gap voltage), shown by the dashed line in the right half (b) of the figure, since the filled states on the left hand side of the junction are now degenerate with the unfilled states on the right hand side. However, if a photon field is applied at frequency $\nu$, increases in tunneling current occur at voltages 
nh $\nu / e$ above and below the gap voltage, due to the photon assisted tunneling process depicted in (a). The resulting (I-V) characteristics show the DayemMartin steps as the full line in (b). This quantum-mechanical detection process is somewhat analogous to optical photodetection and can be used for direct detection or heterodyne detection. The physical structure of an SIS detector is very simple. It is just a small overlap region between the two superconducting electrodes, separated by a thin layer of insulator. The actual structure in practice depends on the style of junction and the nature of the materials used. For the junctions used in early receivers, the electrodes were made from a lead, indium and gold alloy and the insulator was a natural oxide of indium. The structure used to form a junction was usually a shadow-edge overlap region allowing an area of overlap of less than 1 square micron. Such a structure is shown edge-on in figure 2.

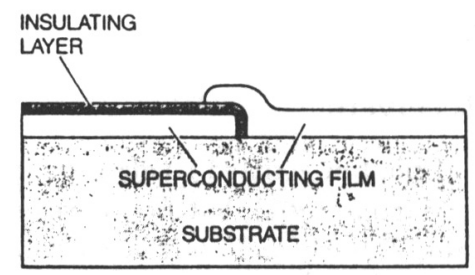

Figure 2: The structure of a simple overlap SIS junction.

For recent junctions with niobium electrodes the fabrication is rather different, starting from a trilayer sandwich of two niobium layers separated by an oxidized aluminum layer (Huggins \& Gurvitch,1985). This is then processed lithographically to leave only a small area of the top electrode. This small remaining top electrode is later contacted by a further superconducting layer to make a circuit. These junctions usually have excellent I-V characteristics, as shown in figure 3 , which depicts the result from a 2.3 square micron $\mathrm{Nb} / \mathrm{Al}_{2} \mathrm{O}_{3} / \mathrm{Nb}$ junction (Zmuidzinas \& LeDuc, 1992).

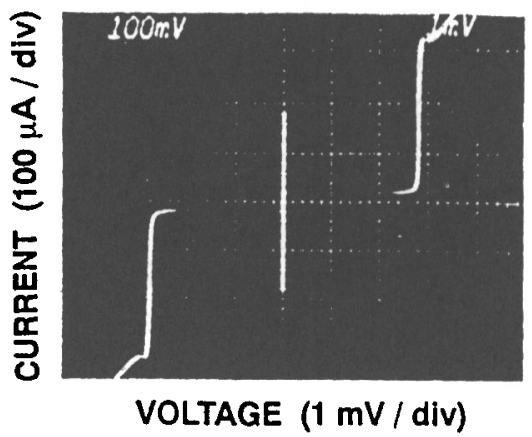

Figure 3: $\mathrm{I}-\mathrm{V}$ characteristics for $\mathrm{a} \mathrm{Nb} / \mathrm{Al}_{2} \mathrm{O}_{3} / \mathrm{Nb}$ junction.

Clearly visible in figure 3 is the zero voltage Josephson current. Most effects of both the AC and DC Josephson effects can be suppressed by application of 
a magnetic field. This is necessary because at high frequencies the Josephson oscillations can interfere with the quasiparticle effect and can cause spurious responses and excess noise. A typical response of a fast modern junction to input power is shown in figure 4 . Here the I-V curve is plotted without LO power, showing the suppression of the Josephson current by magnetic field and the I-V curve with LO power applied at about $500 \mathrm{GHz}$. Only about $10^{-7}$ watts is incident on the junction.

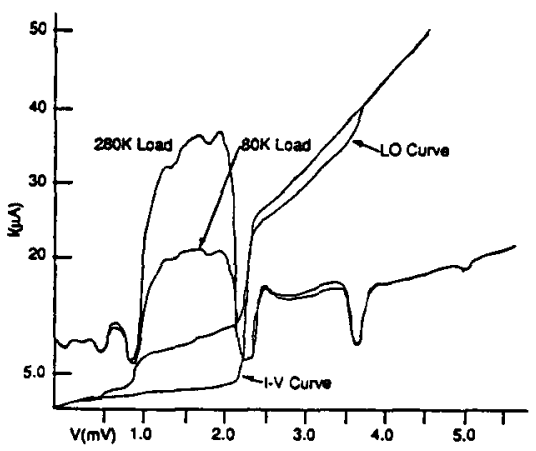

Figure 4: I-V characteristics, with and without $\mathrm{LO}$ power for a $\mathrm{Nb} / \mathrm{Al}_{2} \mathrm{O}_{3} / \mathrm{Nb}$ receiver. Response at $500 \mathrm{GHz}$ to $280 \mathrm{~K}$ and $78 \mathrm{~K}$ loads is also shown. (after Walker et al., 1992)

\section{B SIS Receiver Types}

There are two basic type of SIS receivers, waveguide and quasi-optical. Generally, though not necessarily, waveguide mounted receivers give the best noise performance and are used for single element receivers. Quasi-optical receivers are simpler to construct for high frequencies and are more suitable for array applications. In principle they have larger intrinsic bandwidth, but may be more suscep tible to saturation.

There are too many examples of waveguide SIS receivers to discuss them all. Some examples are the reduced height versions in use at AT\&T Bell Labs, NRAO and IRAM and full height versions at the CSO, SRON and Arizona. Generally, reduced height waveguide allows better matching of the detectors, but full height waveguide is easier to deal with at submillimeter wavelengths. Traditionally waveguide receivers use one or two tuners to help match the detector impedance to the waveguide over much of the waveguide band. The idea is to transform the real and imaginary components of the impedance from quite outlying parts of the Smith chart, back to a nearly real and convenient $(50-100 \Omega)$ values. Examples are receivers due to Kerr \& Pan, 1990, Blundell et al., 1988, Ellison \& Miller, 1987, Kooi et al., 1991 Walker et al., 1992; DeLange et al., 1992 and Honingh et al., 1992. The structure used by Ellison at the CSO is shown in figure 5. This design follows the concepts used earlier for Schottky mixers. It uses a scalar feed to couple both signal and LO radiation via a transformer section to rectangular full height waveguide. The junction is mounted across the guide in the strong E-field. It is preceeded by an E-plane tuner and followed by a backshort. The 
CSO receivers constructed by Kooi and Walker are essentially scaled from this design.

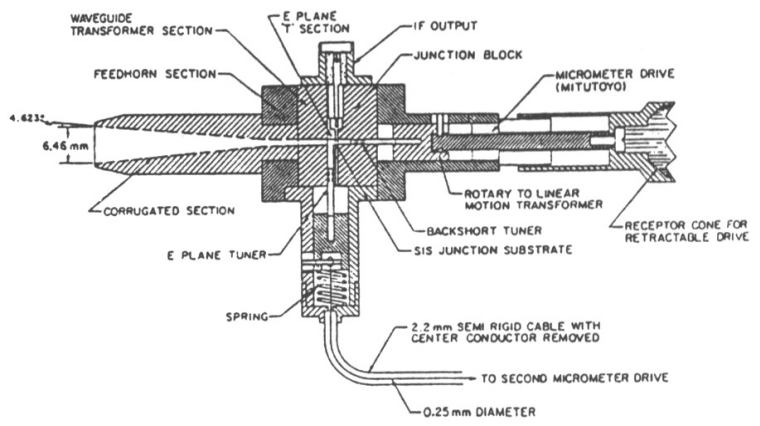

Figure 5: A schematic view of the two-tuner full height waveguide receiver design in use at the CSO for the $200-300 \mathrm{GHz}$ band.

An alternative to the waveguide tuner concept is a design which employs lithographic tuning, in the form of an inductive microstrip stub, actually on the detector chip. This is clearly superior, since it avoids the mechanical complexity of the tuner and puts the reactances where they should be, as close as possible to the detector element. Excellent performance has been achieved in the 100 to $300 \mathrm{GHz}$ range with such a concept by Kerr et al., 1992. In fact these "tunerless" mixers perform just as well as the best tuner-mount mixers. Of course combinations of on-chip tuning and waveguide tuners can also be used.

A recent development is the quasi-optical SIS receiver. The idea here is to generate an integrated SIS junction and lithographic antenna structure, which replaces the entire feed horn, waveguide/tuners and SIS junction chip combination. Ideally the all-planar structure should be well coupled to the input radiation from the telescope. The first attempt at such a quasi-optical SIS receiver was that of Wengler et al., (1985), which used a hyper-hemispheric lens coupled to a bow-tie, planar antenna. While this receiver had very encouraging noise temperatures and worked to very high frequency $(750 \mathrm{GHz})$, it did not have a very good forward beam pattern. The optical scheme is shown in figure 6. An improvement on the bow-tie antenna was the spiral pattern antenna used by Büttgenbach et al., (1988). Here a logarithmic spiral pattern is formed to replace the bow-tie. This has a much improved forward beam pattern and it was found that if the hyper-hemispheric lens were replaced by an elliptical lens with the antenna near the second focus, a much narrower forward beam was formed (Büttgenbach et al. 1993). The plastic lens is then not needed. This receiver has performed well on the telescope at $500 \mathrm{GHz}$ with similar coupling efficiencies to those of waveguide receivers. Like the bow-tie receiver it has a large available bandwidth (200-500 GHz). A further improvement in quasi-optical SIS receivers is the twin-slot dipole-antenna design of Zmuidzinas and LeDuc (1992). The major advantage is that the slot antenna has a very low impedance over a reasonably large frequency range and so couples effectively to large area junctions. It works to high frequencies because a match can be achieved even if the junction capacitance dominates the impedance. This antenna design is also 


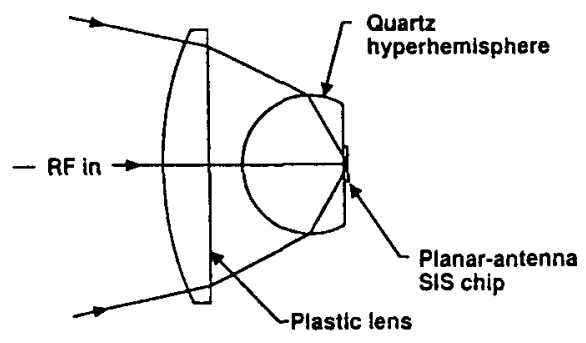

Figure 6: A quasi-optical receiver configuration employing a bow-tie structure and hyper-hemispheric lens. The SIS junction lies at the apex of the bow-tie, itself at the focus of the lens system

used with the hyper-hemispheric lens. In the design of Zmuidzinas and LeDuc the signals are coupled from each slot to the junction by tapered microstrip lines as shown in figure 7. A more recent, multiple junction version of the receiver has given excellent results up to $580 \mathrm{GHz}$ with noise temperatures as low as $110 \mathrm{~K}$ at $500 \mathrm{GHz}$.

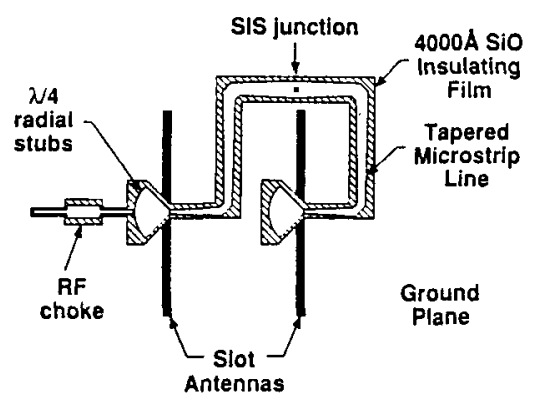

Figure 7: The twin slot planar structure of Zmuidzinas and LeDuc.

As for the waveguide receivers, it is possible to use lithographic tuning structures to match out the capacitance of the junctions. These can be in the form of open-ended microstrip stubs, but it has been shown by Büttgenbach et al. (1992) that a broader band match can be achieved by means of an impedance transformer section in combination with an end-loaded stub. Figure 8 shows the apex area of a spiral antenna with two symmetric matching networks leading away from the apex and two junctions at the ends of the microstrips sections. By means of a Fourier Transform Spectrometer it was shown that this circuit had essentially the theoretically anticipated bandwidth of about $300 \mathrm{GHz}$ centered at about $400 \mathrm{GHz}$. On-chip matching is also used in the latest $\mathrm{Zmuidzinas}$ and LeDuc circuit.

A recent compilation of noise temperatures for SIS and Schottky receivers is shown in figure 9 . It is very encouraging to note that the hoped for performance 


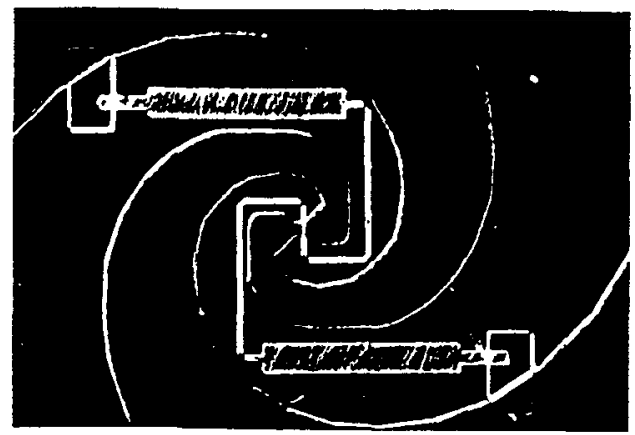

Figure 8: A lithographic matching network near the apex of a spiral antenna.

for SIS devices of about $10 \mathrm{~h} \nu / \mathrm{k}$ is achieved up to $500 \mathrm{GHz}$. At higher frequencies the $\mathrm{Nb}$ SIS receivers grow worse, until at $800 \mathrm{GHz}$ and above the Schottky receivers are better.

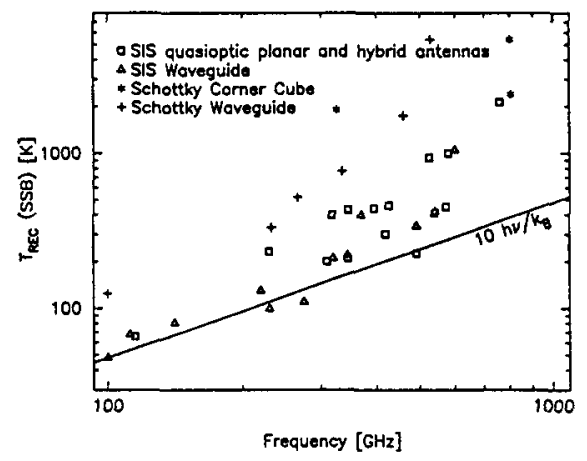

Figure 9: A compilation of receiver noise temperature.

\section{SIS Materials}

According to theoretical expectations the noise performance of SIS receivers should be essentially proportional to frequency up to the gap. Above the gap the noise temperature increases quite rapidly. As a result there is considerable interest in high $2 \Delta$ materials. Lead alloys (eg. $\mathrm{Pb}: \mathrm{In}: \mathrm{Au}$ ) usually have gap frequencies of about $600 \mathrm{GHz}$. (Lead bismuth alloys can be considerably higher, but they are hard to prepare and keep chemically stable). The $\mathrm{Nb} / \mathrm{Al}_{2} \mathrm{O}_{3} / \mathrm{Nb}$ junctions usually have gap frequencies of about $700 \mathrm{GHz}$, which is a little better than lead. Since the Nb based junctions are also chemically more stable than the lead and are better able to withstand thermal cycling, they have taken over as the junctions of choice. However, the problem remains as to what materials to use for frequencies above $700 \mathrm{GHz}$. At the moment most effort is going into niobium-nitride materials. The $\mathrm{Tc}$ values for thin films of $\mathrm{NbN}$ are about $17 \mathrm{~K}$ and the gap voltages of junctions can be in excess of $5 \mathrm{mV}$, so the equivalent frequency may be as high as $1200 \mathrm{GHz}$.

The progress on NbN SIS junctions has been steady, but they still are not 
fully competitive. The best techniques at the moment involve the use of $\mathrm{MgO}$ barriers and heating of the substrate (Shoji et al., 1985; LeDuc et al., 1987) to promote the formation of high quality $\mathrm{NbN}$ layers. Very small area $\mathrm{NbN}$ junctions have been formed by the "edge" process (Hunt et al., 1989). Presumbly progress will be in the area of improved crystallographic quality of the junctions, by means of epitaxial growth techniques.

A final hope for higher $2 \Delta$ materials is the high $T c$ copper-oxide superconductors. However, none of these materials has yet shown tunneling characteristics of the quality or gap definition needed for SIS detectors.

\section{Josephson Mixers}

The Josephson effect involves tunneling of pairs of electrons and, as such, is potentially present in the SIS structure. In the past, the Josephson effect was used for mixing with point contact devices, which were very hard to control and whose parasitic reactances could not easily be determined (eg. Taur \& Kerr, 1978; Claussen \& Richards, 1978). They exhibited "excess noise". The I-V characteristic of the point contact Josephson mixer are those given by the "RSJ" model and are non-hysteretic. When a local oscillator field is applied "Shapiro" steps are seen at zero voltage and above. Now, modern lithography allows the construction of well formed tunnel junctions, but which are hysteretic due to the large capacitances. It is possible to synthesize a Josephson, non-hysteretic junction from an SIS structure by the addition of a lithographic resistor. The question is whether such devices can be used to better control the parameters of the Josephson mixer system and so produce better performance than previously, and possible make use of the high IcRn values of modern (high $\mathrm{Tc}$ ) materials to work at high frequencies. An initial effort is this direction has been carried

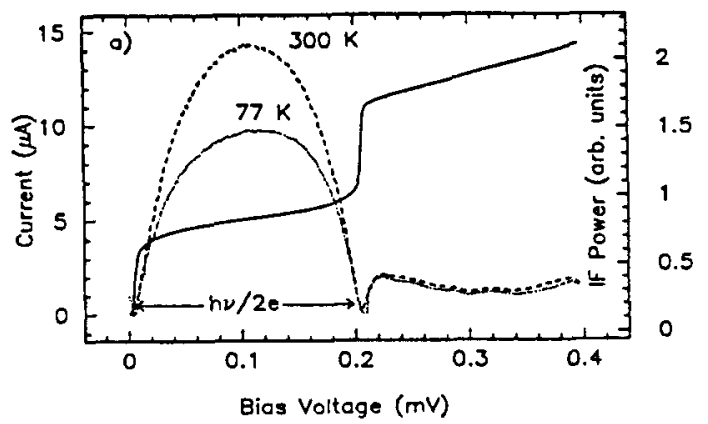

Figure 10: The I-V characteristics and IF power curves for a resistively shunted $\mathrm{Nb} / \mathrm{Al}_{2} \mathrm{O}_{3} / \mathrm{Nb}$ junction of area 0.25 square microns. This test device showed a noise temperature of $390 \mathrm{~K}$ and conversion loss of $6.5 \mathrm{~dB}$ at $100 \mathrm{GHz}$.

out at Caltech. Schoelkopf et al. (1993) have used a Au:Ge resistor across a 0.25 square micron $\mathrm{Nb} / \mathrm{Al}_{2} \mathrm{O}_{3} / \mathrm{Nb}$ junction to synthesize an $\mathrm{RSJ}$ structure. The I-V characteristic and IF power curves for such a device are shown in figure 10. At about $100 \mathrm{GHz}$ this initial, and far from optimum, test device showed a noise temperature of $390 \mathrm{~K}$. Most impressively, however, it is a very stable 
and controllable system and shows very smooth response curves. Efforts will continue to optimize such devices and to test them at higher frequencies.

\section{Conclusions}

Progress in receivers for millimeter and submillimeter astronomy has been very rapid in recent years. Low temperature SIS devices have demonstrated noise temperatures of slightly better than $10 \mathrm{~h} \nu / \mathrm{k}$ or $0.5 \mathrm{~K} / \mathrm{GHz}$ up to $500 \mathrm{GHz}$ and for ground based astronomy this often means that spillover and sky noise may be comparable to receiver noise, so that, in terms of noise temperature, not much more improvement is needed. However, it is likely that these receivers could show lower noise temperatures by a factor of two or three as final optimization takes place. Another area of improvement, as discussed above, will be the increase in upper frequency limit for SIS receivers when $\mathrm{NbN}$ or other high Tc materials are fully developed. Finally, it is now possible to imagine the construction of multibeam SIS receivers of possibly 20 elements or so, since the new quasi-optical planar antenna configurations are easily reproduced and take up very little room in a cryostat.

\section{References}

Blundell, R., Carter, M.J., Gundlach, K.H., 1988, Int. J. IR\&MM Waves 9,361. Blundell, R. \& Tong C.Y.E., 1992, Proc. IEEE 80, 1702.

Büttenbach, T.H., Miller, R.E., Wengler, M.J., Watson, D.M. \& Phillips, T.G., 1988, IEEE Trans. MTT 36, 1720.

Büttgenbach, T.H., LeDuc, H.G., Maker, P.D. \& Phillips, T.G., 1992, IEEE Trans. Appl. Supercond. 2, 165.

Büttgenbach T.H., 1993, IEEE MTT, in press.

Claassen, J.H. \& Richards, P.L., 1978, J. Appl. Phys. 49, 4130.

Crowe, T.W., Mattauch, R.J., Roeser, H.P., Bishop. W.L., Peatman, W.C.B. \& Liu. X., 1992, Proc. IEEE 80, 1827.

Dayem, A.H. \& Martin, R.J., 1962, Phys. Rev. Lett. 8, 246.

DeLange, G. et al. 1992, Proc. 3rd Int. Symp. Space Teraherz Tech.

Dolan, G.J., Phillips, T.G. \& Woody, D.P., 1979, Appl. Phys. Lett. 34, 347.

Ellison, B.N. \& Miller, R.E., 1987, Int. J. IR\&MM Waves 8, 608

Honingh, E.C. et al., 1992, Proc 3rd Int. Symp. Space Terherz Tech.

Huggins, H.A. \& Gurvitch, M., 1985, J. Appl. Phys 57, 2103.

Hunt, B.D., LeDuc, H.G., Cypher S.R. \& Stern, J.A., 1989, Appl. Phys. Lett. $55,81$.

Kerr, A.R. \& Pan, S.K., 1990, Int. J. IR\&MM Waves 11, 1169

Kerr, A.E., Pan, S.K., Lichtenberger, A.W. \& Lea, D.M., 1992, IEEE Mic \& Guided Wave Lett. 2, 1051.

Kooi, J.W., Chan, M., Phillips, T.G., Bumble, B. \& LeDuc, H.G., 1992, IEEE MTT 40, 812.

LeDuc, H.G., Stern, J.A., Thakoor, S. Khanna, S., 1987, IEEE Trans. Mag. MAG-23, 863.

Penzias, A.A., \& Burrus, C.A., 1973. Ann. Rev. Aston. Ap. 11, 51. 
Phillips, T.G. \& Jefferts, K.B., 1973, Rev. Sci. Instr. 44, 1009.

Phillips, T.G. \& Woody D.P., 1982, Ann. Rev. Astron. Ap. 20, 285.

Richards, P.L., Shen, T.M., Harris, R.E. \& Lloyd, F.L., 1979, Appl. Phys. Lett. $34,345$.

Schoelkopf R.J., Phillips, T.G. \& Zmuidzinas, J., 1993, IEEE Trans. Appl. Supercond., in press.

Shoji, A., Aoyagi, M., Kosaka, S., Shinoki, F. \& Hayakawa, H., 1985, Appl, Phys, Lett. 49, 1098.

Taur, Y. \& Richards, P.L., 1978, Appl. Phys. Lett. 32, 775.

Tucker, R.J., 1979, IEEE QE 15, 1234.

Tucker, J.R. \& Feldman, J.J., 1985, Revs. Mod. Phys. 57, 1055.

Walker, C.K., Kooi, J.W., Chan, M., LeDuc, H.G., Carlstrom, J.E. \& Phillips, T.G., 1992, Int. J. IR\&MM Waves 13, 785.

Weinreb, S. \& Kerr, A.R., 1973, IEEE J. Solid-State Circuits 8, 58.

Wengler, M.J., Woody, D.P., Miller, R.E. \& Phillips, T.G., 1985 Int. J. IR\&MM Waves 6, 697 .

Yang, J.X., Agahi, F., Dai, D., Grammer, W., Lau, K.M. \& Yngvesson, K.S., 1991, Semicond. Device Symp. U. Virginia.

Zmuidzinas, J. \& LeDuc, H.G., 1992, IEEE. Trans. MTT 40, 1797.

Question- K.Y. Lo - Whose work is it on the Josephson effect mixer?

Answer - This is the work of Robert Schoelkopf, a graduate student at Caltech.

Question- K.Y. Lo - $\mathrm{Nb} / \mathrm{Al}_{2} \mathrm{O}_{3}, / \mathrm{Nb}$ junctions have been successfully made at the University of Illinois since 1988 and tunerless SIS mixers have been made for the BIMA receivers. Also, the twin-slot planar antenna mixer of Jonas Zmuidzinas was developed while he was at the University of Mlinois.

Question- J. INATANI - Do you think that the Josephson effect mixer can realize a better noise performance than the SIS mixer at frequencies higher than $500 \mathrm{GHz}$ ?

Answer - Possibly, in time. The predicted noise temperature for Josephson effect mixers is a few times the ambient temperature (in the RSJ model). If the IcRn product is large enough the Josephson effect mixer in principle outperforms the SIS mixer near, or above the gap frequency. 